\title{
The Radio and X-Ray Association in Massive Jet Ejection Events of GRS 1915+105
}

\section{Masaaki Namiki ${ }^{* a}$, Sergei A. Trushkin ${ }^{b}$, Taro Kotani $^{c}$, Nobuyuki Kawai ${ }^{c}$, Nicolai N. Bursov ${ }^{b}$, and Sergei N. Fabrika ${ }^{b}$}

a Osaka University, 1-1 Machikaneyama, Toyonaka, Osaka 560-0043, Japan

${ }^{b}$ Special Astrophysical Observatory, Nizhnij Arkhyz, Karachaevo-Cherkassia 369167, Russia

${ }^{c}$ Tokyo Tech, 2-12-1 O-okayama, Meguro, Tokyo 152-8551, Japan

E-mail: namiki@ess.sci.osaka-u.ac.jp

The association between radio and X-ray activities in flaring events of microquasar GRS $1915+105$ is studied. With the RATAN-600 radio telescope, the light curves at 1.0, 2.3, 4.8, 7.7, and $11.2 \mathrm{GHz}$ have been measured in the monitoring program from 15 September to 15 November 2005, and seven bright flaring events were detected in October. Using the ASM/RXTE data, it is clearly shown that all the radio flare events have X-ray counterparts. The X-ray events are relatively bright up to 2 Crabs at $2-12 \mathrm{keV}$. The X-ray light curves show a significant softening of the hardness ratio during the radio flares by a fraction of $\sim 30 \%$. It is found the X-ray spikes accompanying a strong radio flares are different from those without jets, and only such special X-ray spikes may correlate with "massive jet ejections".

VI Microquasar Workshop: Microquasars and Beyond

September 18-22 2006

Società del Casino, Como, Italy

${ }^{*}$ Speaker. 


\section{Introduction}

The X-ray transient source GRS $1915+105$ was discovered in 1992 by Castro-Tirado et al. with the WATCH instrument on board GRANAT. In 1994, a superluminal motion of the jet had been detected from GRS 1915+105 (Mirabel and Rodriguez 1994), since then a new class of astrophysical objects "Microquasar" was established.

Many X-ray observations of GRS 1915+105 revealed remarkable QPOs which are believed to arise in the accretion disk around a black hole. On the other hand, the understanding of the jet phenomena is still quite preliminary, because of the difficulty to detect the X-ray signal of the jet without the effect of the activities of the accretion disk. To interpret the X-ray data correctly, longterm radio monitoring data are desirable. By reference to radio data, the activity of the source can be diagnosed. Furthermore, the massive jet ejection events, by which the source was recognized as a microquasar in the first place, can be predicted by means of radio monitoring. A massive jet ejection event from another microquasar, SS 433, was observed with RXTE with the help of radio monitoring (Kotani et al. 2006).

In this paper, ASM/RXTE data of GRS 1915+105, during radio fhres observed with RATAN600 , are analyzed. Radio fhres successively occurred in the end of October 2005. The ASM X-ray data during the radio fhres were chosen for our analysis. The radio and X-ray simultaneous data reported here provide the jet activity with least effect of the accretion disk. This will allow us to study of the jet itself.
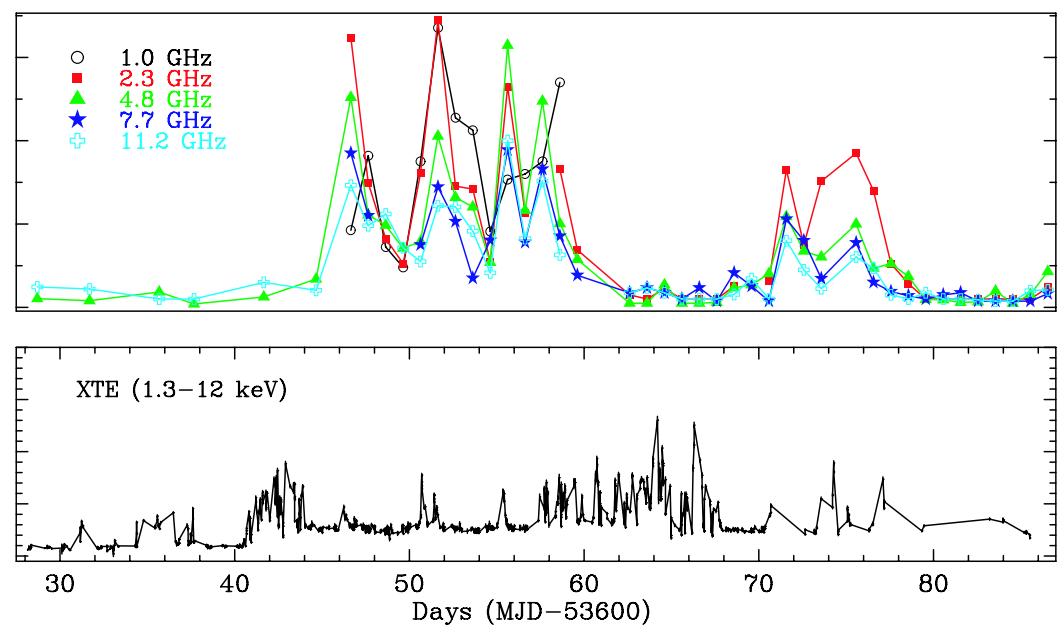

Figure 1: Light curves of GRS 1915+105 obtained with RATAN-600 and ASM/RXTE

\section{Observations}

The RATAN-600 observations have been carried out as a part of a monitoring program of microquasars to study the fhre activity in the wide frequency range. The observations were made using the 'Northern sector' antenna of RATAN-600 radio telescope almost daily from September 2005 to January 2006. The receivers at 1.0, 2,3, 4.8, 7,7 11.2, and 21.7 GHz were used. 
In the X-ray band, the publicly available light curves of GRS 1915+105 ASM on board RXTE have been analyzed. The definitive versions of the dwell by dwell data products in three ASM energy bands, roughly corresponding to $1.3-3,3-5$ and 5-12 keV, were used for our analysis. The results provided by the ASM/RXTE teams at MIT and at the RXTE SOF and GOF at NASA's GSFC.

\section{Results}

\subsection{RATAN-600 and ASM/RXTE light curves}

Figure 1 shows light curves of GRS $1915+105$ obtained with RATAN-600 at 1.0, 2.3, 4.8, 7.7, and 11.2 GHz (top), and obtained with ASM/RXTE at 1.3-12.0 keV (bottom). The seven radio fhres and corresponding X-ray spikes are clearly detected.

\subsection{Radio spectra of flares}
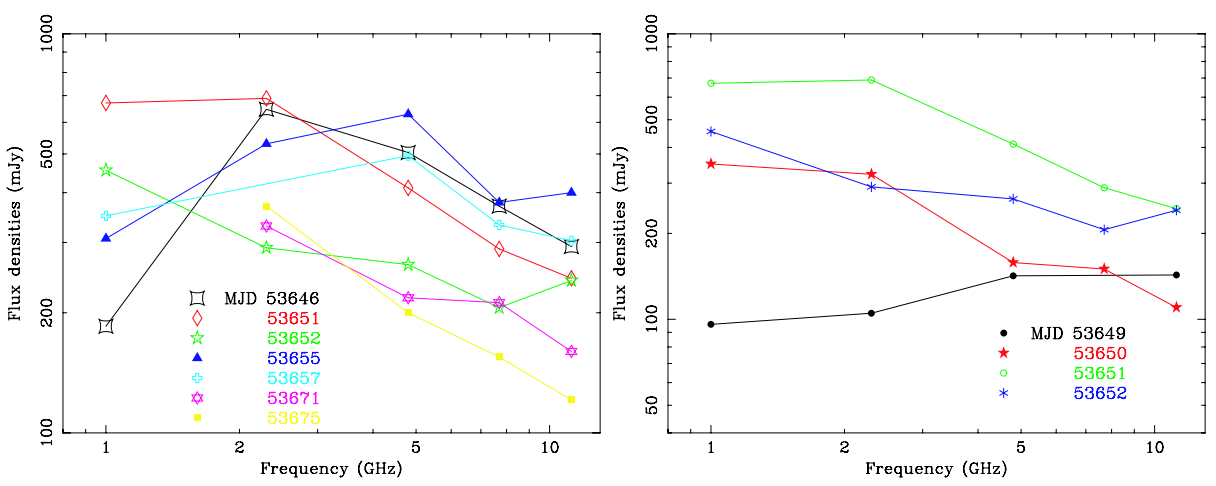

Figure 2: Radio spectra of seven flares at the peak (left), and around the peak of MJD 53651 event (right).

In figure 2, radio spectra of the seven flares at the peak are shown in the left panel. Most of the flares show steep features with spectral indices of $\sim-0.6$. The right panel of figure 2 shows the radio spectra around the peak of the event on MJD 53651. The spectra became steeper with the approach of the peak of the fhre, as the spectral index changed from 0.1 to -0.4 .

\subsection{X-ray profiles}

Figure 3 shows X-ray light curves of GRS $1915+105$ at 1.3-5.0 keV (upper) and 5.0-12.0 $\mathrm{keV}$ (middle), and hardness ratio (bottom) during the same span as figure 1. The green vertical dashed lines indicate the X-ray spikes corresponding to the seven radio fares in figure 1 . The black horizontal dashed line in the bottom panel shows the average values of the hardness ratio.

Figure 4 shows the X-ray light curves (1.3-12.0 keV; top) and the hardness ratio (bottom) of around the peak on MJD 53646(left), MJD 53651 \& 53652 (center), and MJD 53655 (right). The black dashed lines show the average values in the range of each figure. The profiles of the X-ray spikes during the radio fhres are clearly distinguishable from other spikes because of their shape, which shows a fast rise and exponential decay. The other X-ray spikes, which reffect the activity of 


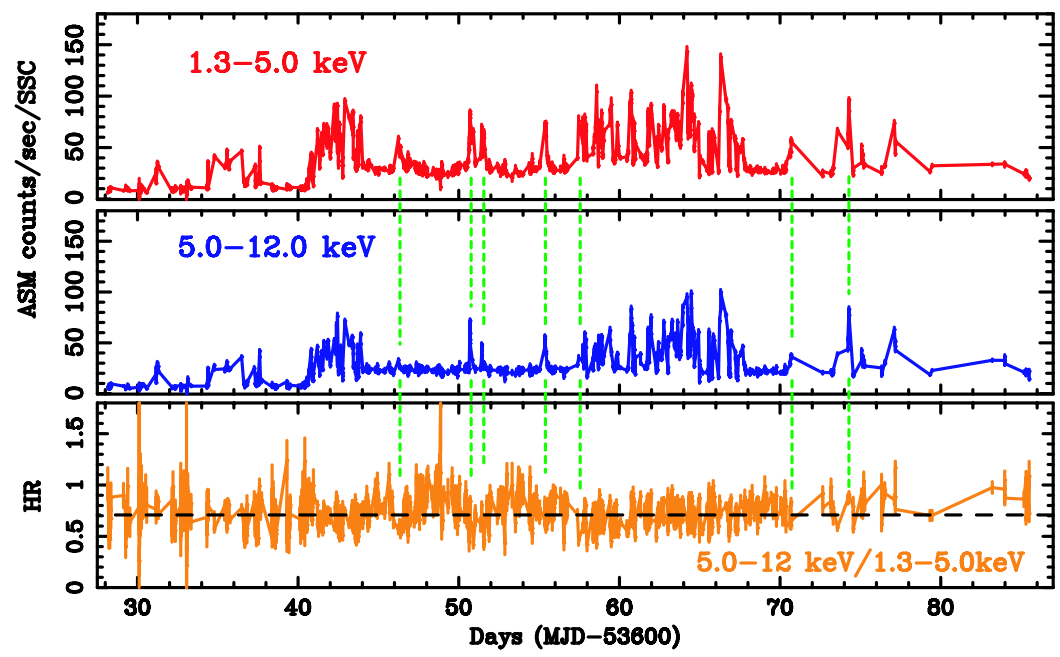

Figure 3: X-ray light curves at 1.3-5.0 keV (upper) and 5.0-12.0 keV (middle), and hardness ratio (bottom).
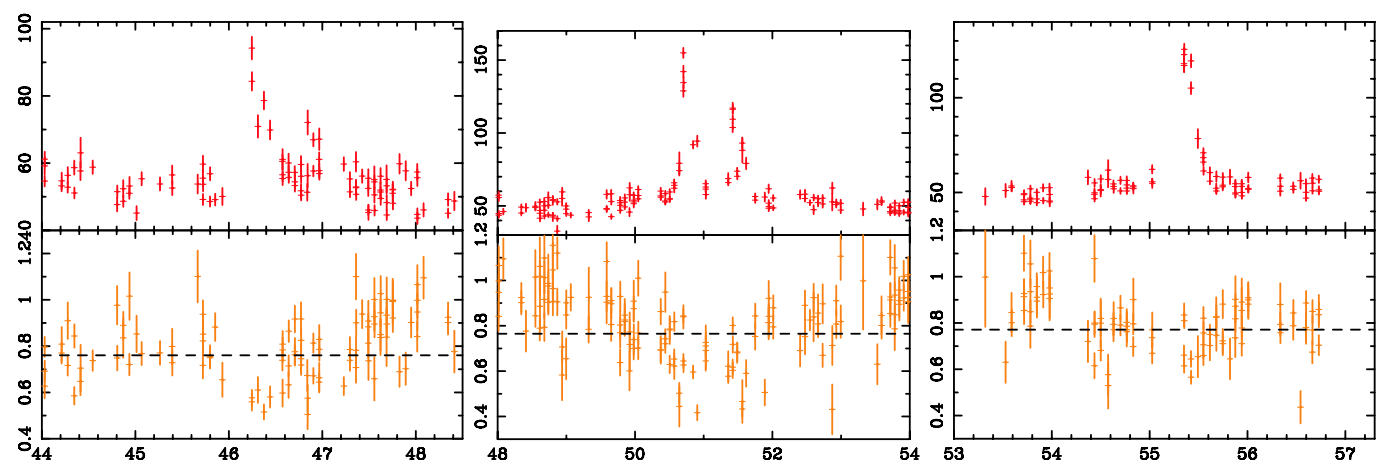

Figure 4: X-ray light curve hardness ratio of the around the peak of the flares.

the accretion disk, show an irregular pattern. During the radio fhre, the spectra of the X-ray spikes become softer than those of the quiescent phase, by a fraction of $\sim 30 \%$.

\section{Summary and Discussion}

The analysis of the RATAN-600 and ASM/RXTE data of the GRS 1915+105 in the active phase on October 2005 has yielded the following results:

- There are clear relations between the radio fares and X-ray spikes, it will allow us to extract the jet component without that of the other component.

- The radio spectra become steeper with the approach of the peak of the fhre.

- The profiles of the X-ray spikes during the radio fares show a fast rise and exponential decay, which is different from the other activities, such as those of the accretion disk. 
- The X-ray light curves show a softening (in 1.3-5.0keV/5.0-12.0 keV ratio) during the radio flares by a fraction of $\sim 30 \%$.

- The X-ray spike accompanying a strong radio fare has special characteristics.

- Only these special X-ray spikes might eject the blob (= jet, radio flare).

The simultaneous radio and X-ray monitoring is very valid for the investigation of the massive jet ejection event from microquasars. Future observations of massive jet ejections from microquasars are encouraged.

Acknowledgments. These studies are supported by the Russian Foundation Base Research (RFBR) grant N 05-02-17556, the mutual RFBR, and Japan Society for the Promotion of Science (JSPS) grant $\mathrm{N}$ 05-02-19710.

\section{References}

[1] Castro-Tirado, A. J., Brandt, S., \& Lund, N., IAUC, 1992, 5590

[2] Mirabel, I. F., \& Rodriguez, L. F., 1994, Nature, 371, 46

[3] Kotani, T., Trushkin, S. A., Valiullin, R., Kinugasa, K., Safi-Harb, S., Kawai, N., \& Namiki, M., ApJ, 2006, 637, 486 